CASE REPORT

\section{E. Dugert}

S. Lagleyre

A. Brouchet

O. Deguine

C. Cognard

F. Bonneville

\title{
Osteoid Osteoma Invading the Posterior Labyrinth of the Petrous Bone
}

SUMMARY: We report a painless osteoid osteoma of the inner ear in an elderly woman presenting with hearing loss, tinnitus, and vertigo. CT showed a heterogeneous mass containing calcifications involving the posterior labyrinth, which heterogeneously enhanced on postcontrast MR images. Osteoid osteoma should be included in the differential diagnosis of an osteolytic petrous bone lesion with central calcification and enhancement.

ABBREVIATIONS: $\bigcirc O$ = osteoid osteoma

$\mathbf{0}$ steoid osteoma is a benign osteoblastic tumor, most frequently diagnosed in young men presenting with characteristic pain at the site of the lesion, increasing at night and relieved by salicylates. On CT, the lesion is characterized by a zone of bony sclerosis surrounding a central radiolucent area. ${ }^{1}$ OO usually involves long bones and the vertebral column and extremities and is very uncommon in the skull. ${ }^{2}$ Temporal bone $\mathrm{OO}$ is even rarer, with only 4 cases described in the literature, none of which were located in the inner ear. ${ }^{3-5}$ We report here the first case of a pathologically proved $\mathrm{OO}$ invading the posterior labyrinth and review the relevant literature.

\section{Case Report}

A 77-year-old healthy woman presented with a several-months' history of right hearing loss, vertigo, and tinnitus. There was no associated pain. Physical examination confirmed hearing loss and an estimated vestibular deficiency of $70 \%$ on the right side. CT showed an osteolytic, heterogeneous, rounded, well-demarcated petrous lesion, measuring $11 \times 6 \mathrm{~mm}$, with central calcifications (Fig $1 A$ ). Although such calcifications have been interpreted as newly produced osteoid trabeculae, remnants of destroyed bone might have been possible as well. The lesion spared the cochlea but invaded the posterior labyrinth and the endolymphatic duct. There was a close relationship with the facial nerve (Fig 1B). No sclerotic bone was seen surrounding the lesion. At MR imaging, the lesion demonstrated intermediate signal intensity on T1 and high signal intensity on T2-weighted images and enhanced intensely and heterogeneously after intravenous gadolinium administration (Fig 2). Technetium Tc99m scintigraphy showed a corresponding increased uptake in the petrous bone. The preoperative diagnosis was hemangioma.

A surgical approach through a mastoidectomy revealed a gray lesion. The center of the lesion was soft and hemorrhagic, but its periphery was hard and indistinguishable from the surrounding normal bone. Because of the anatomic contact with the tympanic segment of the facial nerve, the lesion was only partially excised. Definitive pathologic examination revealed a nidus formed by a hypervascular young conjunctival stroma with osteoblasts, multinucleated giant cells, and thin osteoid trabeculae, consistent with osteoid

Received July 12, 2009; accepted after revision September 7.

From the Departments of Neuroradiology (E.D., C.C., F.B.), Otorhinolaryngology (S.L., O.D.), and Histopathology (A.B.), University Hospital Center of Toulouse, Purpan Hospital, Toulouse cedex, France.

Please address correspondence to Pr. Fabrice Bonneville, MD, PhD, Department of Neuroradiology, Purpan Hospital, Place du Docteur Baylac, TSA 40031, 31059 Toulouse cedex 9, France; e-mail: fabrice.bonneville@gmail.com

DOI 10.3174/ajnr.A1910 osteoma (Fig 3). The postoperative course was uneventful, with normal facial nerve function. At 6-month follow-up, the vertigo had disappeared but the tinnitus remained unchanged.

\section{Discussion}

$\mathrm{OO}$ is a frequent, benign, primary bone tumor accounting for approximately $10 \%$ of all primary bone tumors. ${ }^{1,2}$ It is most frequently seen in teenagers and young adults and is very rare after 30 years of age. Although our patient was a woman, OO usually prevails in men with a sex ratio of 2:1. Moreover, the location of the lesion in the inner ear is unique because $\mathrm{OO}$ mostly involves long bone diaphysis, extremities, and the vertebral column. Temporal bone OO has previously been reported in the mastoid and the middle ear ${ }^{3-5}$ but, to the best of our knowledge, never in the labyrinth.

Pain is the main presenting symptom of OO. It usually increases during the night and is relieved by salicylates. The lack of pain with $\mathrm{OO}$ is uncommon. It was reported in only $1.6 \%$ in a large review ${ }^{6}$ and was attributed to the lack of a hard shell or nerve endings around the lesion. In our case, the lack of nerve endings in the otic capsule could explain this unusual clinical feature. ${ }^{7}$ Vertigo, tinnitus, and hearing loss described in our patient might be related to the particular location of the tumor. Indeed, these symptoms may be related to the posterior labyrinth involvement and especially the endolymphatic duct.

On CT, OOs typically demonstrate fusiform sclerotic cortical thickening in the shaft of a long bone. A characteristic radiolucent area, measuring $<1 \mathrm{~cm}$ in diameter and representing the lesion itself, is usually located within the center of the area of osteosclerosis and harbors central calcification in approximately $50 \%$ of cases. ${ }^{2,5,8}$ The lesion in our patient showed such a radiolucent area, with partial central calcification, but no peripheral sclerotic bone was clearly conspicuous because it may have been lost in the high attenuation of the otic capsule.

The presence of a radiolucent lesion with internal calcifications and close contact with the facial nerve also raises the possibility of a temporal bone hemangioma. Although the topography was very unusual for the latter (hemangioma is usually centered near the geniculate ganglion or the internal auditory canal) and no facial nerve palsy was observed, the overall imaging pattern is almost indistinguishable between the entities and final diagnosis is determined by histology.

At MR imaging, the lesion demonstrated intermediate signal intensity on T1-weighted images and high signal intensity on T2-weighted images and heterogeneously enhanced after 

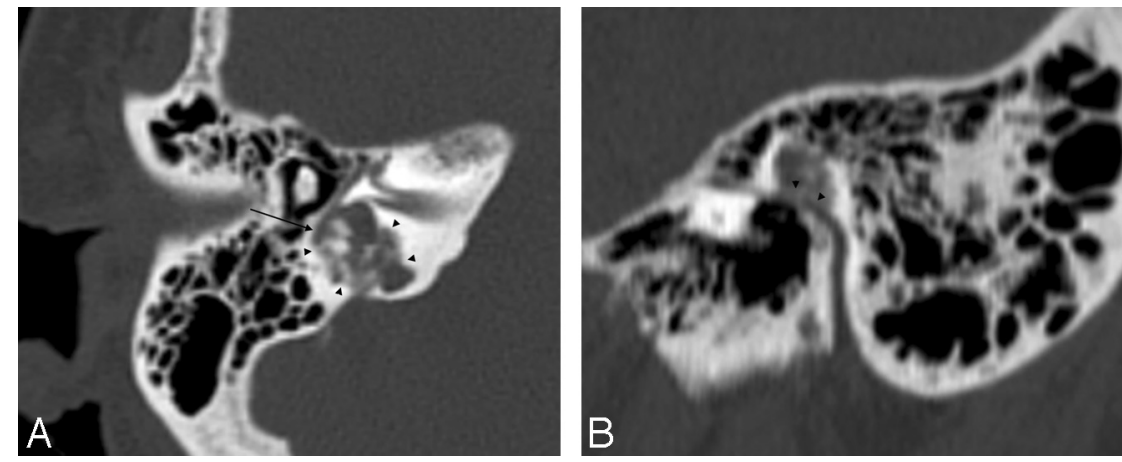

Fig 1. A, Axial CT scan shows an osteolytic heterogeneous mass, with central calcifications invading the posterior labyrinth (arrowheads). Note the close relationship between the second portion of the facial nerve and the tumor (arrow). B, Sagittal CT scan reformat shows a close relationship (arrowheads) between the tympanic segment of the facial nerve and the tumor
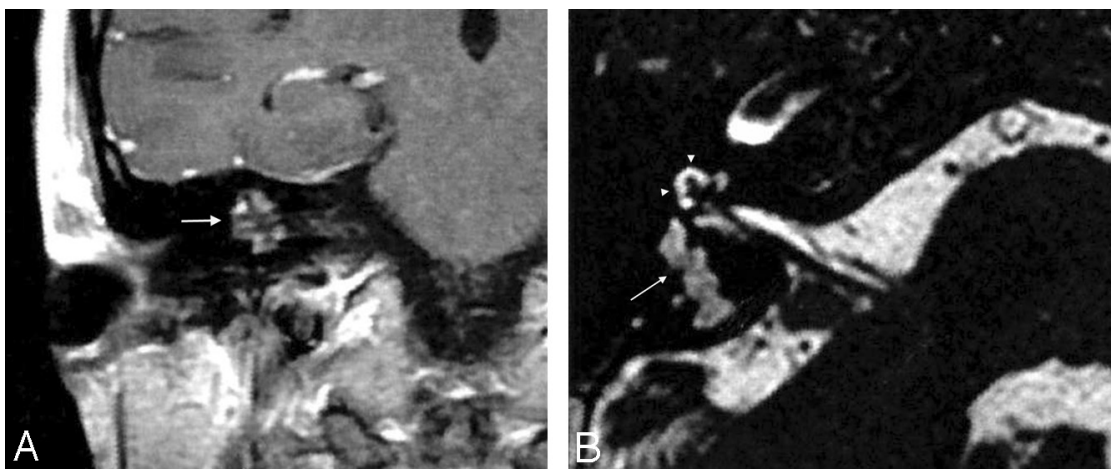

Fig 2. A, Coronal contrast-enhanced T1-weighted image shows heterogeneous enhancement of the right petrous bone lesion (arrow). B, Axial heavily T2-weighted image demonstrates the high-signal-intensity lesion involving the posterior labyrinth (arrow). Note the normal high signal intensity of the cochlea (arrowheads).

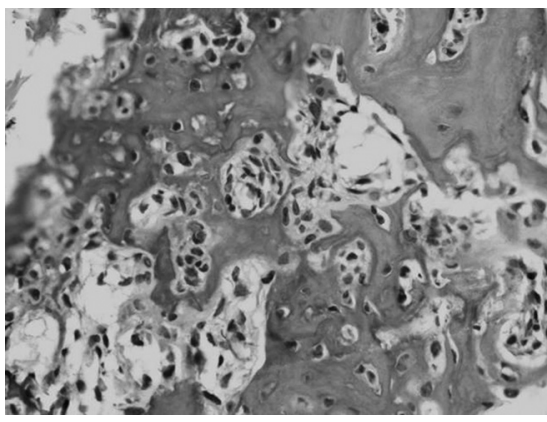

Fig 3. Photomicrograph shows a nidus formed by a hypervascular conjunctival stroma with multinucleated giant cells and thin osteoid trabeculae lined by plump osteoblasts (hemotoxin-eosin, original magnification $\times 200$ ).

gadolinium injection. Endolymphatic sac tumors may involve this region and appear as a heterogeneously enhancing osteolytic tumors arising from the endolymphatic duct. However, endolymphatic sac tumors usually erode the posterior aspect of the petrous pyramid and extend into the cerebellopontine angle cistern. Additionally, at MR imaging, endolymphatic sac tumors are heterogeneous, demonstrating areas of T1 and T2 high signal intensities corresponding to cysts filled with blood or high proteinaceous fluid and multiple small signal intensity voids due to calcifications and blood vessels. ${ }^{9}$

Other considerations for enhancing petrous bone lesions include meningioma, eosinophilic granuloma, glomus tumor, cartilaginous tumors, chordoma, and metastasis, but these are usually larger when symptomatic. ${ }^{10}$ The size of the lesion is an important criterion because it also helps in distinguishing $\mathrm{OO}$ from osteoblastoma, another osteoid-producing tumor. This distinction can be very challenging, even on histology ${ }^{11}$ because both lesions harbor a hypervascular young conjunctival stroma, which contains osteoblasts and multinucleated giant cells in thin osteoid rows that can be calcified. ${ }^{2,4}$ In equivocal cases, the size of the lesion measured on CT remains the most important criterion: OOs are typically $<1 \mathrm{~cm}$, whereas osteoblastomas are $>2 \mathrm{~cm}^{11}$ Another finding that can distinguish the 2 entities in theory is central calcification, because $\mathrm{OO}$ usually contains a single calcification, whereas an osteoblastoma contains multiple calcifications. ${ }^{11}$ Therefore, our case could have been labeled osteoblastoma, but it did not meet the size criteria. In fact, all osteoblastomas of the temporal bone reported in the English literature were noticeably larger than $2 \mathrm{~cm} .{ }^{12}$

It is important to differentiate $\mathrm{OO}$ from osteoblastoma because they have different behaviors and treatments. Generally regarded as "benign," osteoblastomas have definite potential for aggressive behavior, local recurrence, and malignant transformation, while OOs do not.

\section{Conclusions}

We report an unusual case of an osteoid osteoma of the inner ear in an elderly woman who had hearing loss, tinnitus, and vertigo without pain. Although the definitive diagnosis requires histologic confirmation, osteoid osteoma should be included in the differential diagnosis of an osteolytic temporal lesion with sharp margins, central calcification, and enhancement after contrast administration.

\section{Acknowledgment}

We thank Gul Moonis, MD, for her pertinent review and editing of this manuscript. 


\section{References}

1. Resnick D, Kyriakos M, Greenway GD. Tumors and tumor-like lesions of bone: imaging and pathology of specific lesions. In: Resnick D, ed. Bone and Joint Imaging. Philadelphia: Saunders; 1989:1107-81

2. Kransdorf MJ, Stull MA, Gilkey FW, et al. Osteoid osteoma. Radiographics 1991;11:671-96

3. Wilder WM, Dowling EA, Brogdon BG. Osteoid osteoma of the mastoid tip. Skeletal Radiol 1995;24:551-52

4. Pardal Refoyo JL, Ursua Sarmiento I, Benito Alba F. Osteoid osteoma of the temporal bone (mastoid): report of one case [in Spanish]. Acta Otorrinolaringol Esp 1995;46:215-17

5. Iffenecker C, Rocher P, Rabia MH, et al. Osteoid osteoma of the petrous bone. Neuroradiology 1997;39:821-23

6. Jackson RP, Reckling FW, Mants FA. Osteoid osteoma and osteoblastoma: similar histologic lesions with different natural histories. Clin Orthop Relat Res 1977:303-13

7. Schulman L, Dorfman HD. Nerve fibers in osteoid osteoma. J Bone Joint Surg Am 1970;52:1351-56

8. Assoun J, Richardi G, Railhac JJ. Osteoid osteoma: MR imaging versus CT. Radiology 1994;191:217-23

9. Mukherji SK, Albernaz VS, Lo WW, et al. Papillary endolymphatic sac tumors: CT, MR imaging and angiographic findings in 20 patients. Radiology 1997; 202:801-08

10. Mafee MF, Valvassori GE, Becker M. Imaging of the Head and Neck. Stuttgart, Germany: Thieme; 2005: 95-108

11. Kroon HM, Schuurmans J. Osteoblastoma: clinical and radiologic findings in 98 new cases. Radiology 1990;175:783-90

12. Doshi SV, Frantz TD, Korol HW. Benign osteoblastoma of the temporal bone: case report and literature review. Am J Otolaryngol 2001;22:211-14 